\title{
The Lieb-Mattis theorem revisited
}

\author{
W. Florek \\ Adam Mickiewicz University, Faculty of Physics \\ ul. Umultowska 85, 61-614 Poznań, Poland \\ wojciech.florek@amu.edu.pl
}

PACS 75.10.Jm, 75.50.Xx, 03.65Aa, 03.65.Fd

DOI 10.17586/2220-8054-2018-9-2-196-205

\begin{abstract}
Simple formulation and a straightforward proof of the Lieb-Mattis theorem (LMT) do not indicate how powerful a tool it is. For more than fifty years, this theorem has been mainly discussed in its 'strong' form and applied mainly to many type of infinite spin lattices. It can be easily proved that in such cases, geometrically frustrated systems have to be excluded. However, it has been recently shown that the so-called 'weak' or general form of the LMT can be exploited considering some small, geometrically frustrated quantum spin systems. Moreover, many systems, which do not satisfy assumptions of the LMT, show identical features, including the level order characteristic for bipartite spin systems. It yields a question about possible generalizations or modifications of this theorem to involve a larger class of problems. To begin, algebraic aspects have to be understood with the invaluable role of the Perron-Frobenius theorem. The latter theorem is investigated, discussed, modified etc. in immense number of works. Many of them are important in physical applications and ways of reasoning they present can be exploited in different approaches to the LMT.
\end{abstract}

Keywords: quantum spin systems, Lieb-Mattis theorem, level order,ground state properties.

Received: 27 December 2017

Revised: 7 January 2018

\section{Introduction}

Physics and chemistry, both experimental and theoretical, of magnetic nanosystems have experienced rapid progress since pioneering papers on molecular magnets at the turn of the 20th and 21st centuries [1-4]. Among others, this is caused by their possible applications in molecular spintronics, magnetic hyperthermia, quantum computing and for high-density information storage or as magneto-optic devices and magnetic refrigerants. It has to be stressed that such objects are also considered as the testbeds for verification of fundamental quantum theories. At present, investigations are focused on both homo- and heterometallic complexes comprising transition and rare earth metal ions. Their most promising low temperature properties depend crucially on their quantum nature, which leads to the discrete energy levels. And properties of the most significant ground-state level are of great importance. Reliable results can be obtained within the frame of the quantum spin models, with the dominant position of large class of Heisenberg-like systems. There are several strict results (e.g. the well-known Bethe Ansatz and its generalizations) and the Lieb-Mattis theorem (LMT) [5] occupies an important place amongst them. Due to its general character it is not applicable to, for example, anisotropic systems, nevertheless it provides a simple and powerful tool to obtain rigorous results.

The Lieb-Mattis [5] theorem was announced in an earlier paper, in which similar reasoning had been applied to an antiferromagnetic linear chain of spin $s=1 / 2$ [6]. Both papers have influenced investigations in condensed matter physics ${ }^{1}$ and are still highly-cited. Their importance goes beyond condensed matter physics, what is confirmed by many databases like Scopus or Web of Science, and in both cases the number of citations per year increases recently ${ }^{2}$. In condensed matter physics, this is clearly related to development in investigation of, to mention only a few, entanglement, topological phases, single molecule magnets and frustration. Examples of other problems discussed with the use of the LMT or the Lieb-Schultz-Mattis theorem can be found in Refs. [7-14]. On the other hand, there are many attempts to extend these theorems (e.g. Refs. [15-18]). It can be shown [19] that the LMT is, in fact, a consequence of the Perron-Frobenius theorem (PFT) [20-24], so possible extensions of the latter one may lead to generalization of the LMT. In the case of bipartite systems, the transformation proposed in Refs. [5,6] yields a Hamiltonian as a Hermitian matrix with non-negative elements, so the PFT can be applied

\footnotetext{
${ }^{1}$ In April 2017, Ref. [6] was included in a virtual issue of Ann. Phys. as one of the Most Cited in Condensed Matter Theory; see: https://www.journals.elsevier.com/annals-of-physics/virtual-special-issues.

${ }^{2}$ According to the Web of Science Core Collection (December, 2017) Ref. [5] was cited about 600 times in total (approx. 11 per year) and 90 times in the last five years (18 per year). These numbers for Ref. [6] are as follows: more than 2300 (about 40 per year) and about 560 (more than 110 per year).
} 
directly. Non-bipartite systems, like polygons with odd number $n>3$ sides and antiferromagnetic coupling of the nearest neighbors, are not bipartite and, after similar transformation, some entries are negative. However, some results [25-27] indicate possibility to consider matrices with some negative elements, so it is excepted that it will give impact for reconsideration of the LMT and allow to extend the class of systems with well-determined ground-state properties.

It has to be pointed out that most problems considered are based on the so-called 'strong' version of the LMT, which, roughly speaking, excludes systems with competing interactions or geometrically frustrated [28]. The 'weak' (or general) form of this theorem allows some additional and relatively weak antiferromagnetic couplings, leading to geometric frustration in systems which are bipartite according to assumptions of the LMT [29,30], so coexistence of geometric frustration and bipartiteness is admissible. However, at the same time, it demands strong long-range couplings, what is rather non-physical in large (infinite) systems. Therefore, this feature can be observed in small systems or systems with special topology. On the other hand, the so-called Lieb-Mattis Level Order (LMLO) is realized in both, 'strong' and 'weak' cases. Moreover, it can be proved (in some cases numerically only) that larger, non-bipartite in general, analogous of bipartite systems still exhibit the LMLO [30-32]. Similarly, this effect is observed for anisotropic systems, but the total spin number $S$ has to be replaced by the total magnetization $M$ [33-36]. Reformulation of the LMT to include all these cases is a challenging task and to complete it, this theorem itself has to be 'revisited' and mathematical background must be meticulously examined. This paper, as its title says, is mainly devoted to the first part of this project with stress on mathematical correctness.

In the following section systems satisfying postulates of the LMT are presented. Next, in Sec. 3, the theorem itself and its mathematical background, including the PFT, are discussed. Some models not satisfying assumptions of the LMT, but showing the same properties as those being its subject, are shortly described in Sec. 4 and some open problems are indicated. The paper ends with overall conclusions in Sec. 5.

\section{The domain of the Lieb-Mattis theorem}

\subsection{The Heisenberg Hamiltonian}

The original paper of Lieb and Mattis starts with the following Hamiltonian:

$$
H=2 \sum J_{i j} \boldsymbol{S}_{i} \cdot \boldsymbol{S}_{j}
$$

the factor 2, irrelevant in proofs, suggests that this sum is taken over different non-ordered pairs $\{i, j\}$, but it was not clearly stated throughout the whole text. Some steps in considerations could be performed properly (or without special assumptions) for finite systems only, so it should be assumed that $1 \leq i, j \leq n$. In this paper, the LMT is discussed in the context of magnetic molecules (finite quantum spin systems), so this restriction does not lead to any constraints. On the other hand, it is very important that this Hamiltonian is isotropic and commutes with the square of total spin operator $\boldsymbol{S}^{2}$, where $S=\sum_{i=1}^{n} \boldsymbol{s}_{i}$, with non-negative eigenvalues less or equal than $S_{\max }\left(S_{\max }+1\right)$ for $S_{\max }=\sum_{i=1}^{n} s_{i}$. With all these comments the system under question can be determined as follows.

For a given $1<n \in \mathbb{N}$ there is set on indices $V=\{j \in \mathbb{N} \mid 1 \leq j \leq n\}$ and to each of them a spin number $s_{j}$, determining a spin operator $\boldsymbol{s}_{j}$, is unambiguously assigned. The set $E^{\prime}$ of all non-ordered pairs $\{j, k\}$ with $\left(\begin{array}{l}n \\ 2\end{array}\right)=n(n-1) / 2$ elements is constructed in the natural way (with the loss of generality $j<k$ can be assumed). A real-valued function:

$$
J: E^{\prime} \rightarrow \mathbb{R}:\{j, k\} \rightarrow J_{j k}=J_{k j}
$$

determines weights of edges (an exchange integral is assigned to each bond) [37]. In spin models, bonds with $J_{j k}=0$ are neglected, so a mathematical object under consideration is a simple (edge-)weighted graph with nonzero weights $J_{j k} \in \mathbb{R}$ and the set of edges $E=\left\{\{j, k\} \in E^{\prime} \mid J_{j k} \neq 0\right\}$; vertex weights $s_{i}$ are irrelevant in this approach. The isotropic Heisenberg Hamiltonian is determined as the following sum over all non-ordered pairs:

$$
H=2 \sum_{j, k=1 \mid j<k}^{n} J_{j k} \boldsymbol{s}_{j} \cdot \boldsymbol{s}_{k}
$$

the 'non-existing' edges with $J_{j k}=0$ have not been excluded in this sum, since they 'disappear' in the natural way, but considering the graph representation of this model, only the edges with non-zero weights are taken into account. 

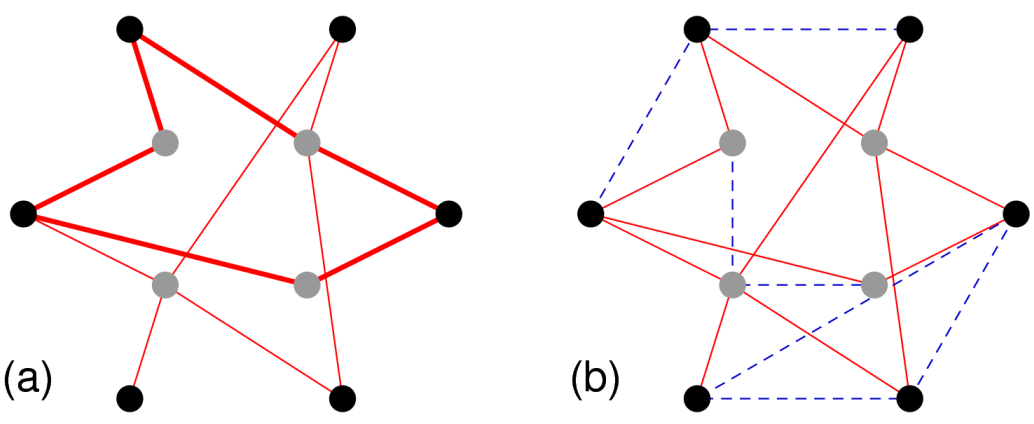

FIG. 1. (a) A bipartite (simple) graph; one of even-length cycles is distinguished by bold lines. (b) A bipartite weighted graph; for a given threshold $t$ solid line edges have weights $w \geq t$, whereas those with weights $w \leq t$ are depicted by dashed lines. In both cases grey and black full circles denote vertices belong to $U$ and $W$ subsets, respectively

\subsection{Bipartiteness}

In graph theory, a graph is bipartite when its set of vertices $V$ can be divided into two disjoint, non-empty and independent sets, i.e. $V=U \cup W, U \cap W=\emptyset, U \cap W=\emptyset$, in such a way that every edge connects vertices from different subsets only [37]. In other words, if $\{j, k\} \in E$, then vertices $j$ and $k$ belong to different sets $U$ and $W$. Equivalently they are determined as graphs with no odd-length cycles, what is a very important property [see Fig. 1(a)].

In graph theory, the matching problem is considered taking into account weights of edges, but a graph in question is still bipartite graph if weights are neglected [37]. It means that the weights of edges do not modify the bipartiteness. The LMT demands rather bipartite weighted graphs than bipartite graphs with weights, i.e. weighted bipartite graphs [37]. In the former case, the bipartiteness is determined taking into account the weights of edges. A formal definition can be stated as follows: A simple edge-weighted graph with non-zero real weights is bipartite with a threshold $t$ if its set of vertices can be decomposed into two disjoint and non-empty sets ( $U$ and $W$, say) such that edges $\left\{u, u^{\prime}\right\}$ and $\left\{w, w^{\prime}\right\}$, where $u, u^{\prime} \in U$ and $w, w^{\prime} \in W$, have weights $w \leq t$, whereas edges $\{u, w\}$ have weights $w \geq t$ [see Fig. 1(b)]. Note that in this case subset $U$ and $W$ are not independent, since vertices from the same set may be adjacent. In the special case $t=0$, when edges with zero weights are omitted, the subgraphs with sets of vertices $U$ and $W$, respectively, have edges with negative weights, whereas existing edges $\{u, w\}$ have positive weights only. Such subgraphs can be named 'negatively weighted subgraphs' and hereafter, in the context of spin systems, they will be referred to as 'ferromagnetic components (parts) of a (spin) system'3. It can be easily seen that in the bipartite weighted graphs with the threshold $t=0$, all cycles contain an even number of edges with positive weights.

The LMT can be applied to spin systems, with the Hamiltonian given by Eq. (1), which can be presented as bipartite weighted graphs, but for $t>0$ they have to be complete ones, which means that for each pair of vertices $u \in U$ and $w \in W$, there exist the edge $\{u, w\}$ with non-zero weight $w \geq t>0$. Due to the inequality signs, when weights are compared with the threshold, the bipartition of a spin system may be not unique and possible thresholds leading to the same bipartition, belong to some, finite or even infinite, range. Using symbols introduced by Lieb and Mattis, the definition of a bipartite (in sense of the LMT) spin system with $n$ spins coupled to each other according to Eq. (1) says: There exists a constant $g^{2} \geq 0$ that indices $1 \leq j \leq n$ can be divided into two subsets $A$ and $B$ with

$$
J_{j j^{\prime}}, J_{k, k^{\prime}} \leq g^{2}, \quad J_{j, k} \geq g^{2}
$$

where $j, j^{\prime} \in A$ and $k, k^{\prime} \in B[5,28]^{4}$; such systems are referred to as LM-bipartite (LMB systems, for short). The completeness of the appropriate graph means that for $g^{2}>0$ even distant spin have to be coupled antiferromagnetically with $J \geq g^{2}$, what is rather non-physical assumption. Therefore, LMB spin systems with $g^{2}>0$ have to be rather small ones or have special topology like centered polygons or pyramids, as presented in Fig. 2.

\footnotetext{
${ }^{3}$ In Eq. (1) negative exchange integrals correspond to ferromagnetic couplings.

${ }^{4}$ Many authors apply the original Heisenberg convention, in which antiferromagnetic exchange integrals are negative, then the inequalities in Eq. (2) have to be reversed and the sign of the threshold has to be changed, i.e. $J_{j j^{\prime}}, J_{k, k^{\prime}} \geq-g^{2}$ and $J_{j k} \leq-g^{2}$. In Ref. [28] the sign was not be modified accordingly.
} 


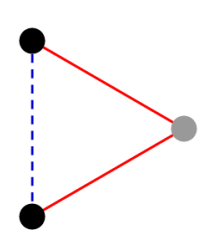

(a)

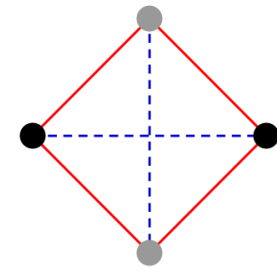

(b)

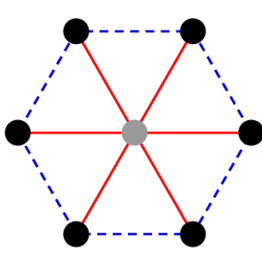

(c)

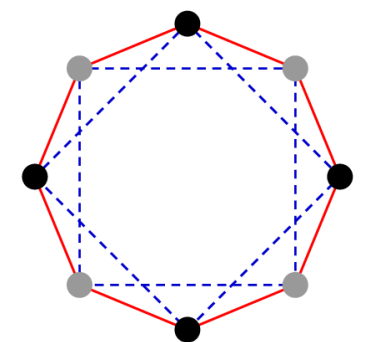

(d)

FIG. 2. The systems (a-c) are LMB ones for any threshold $g^{2}>0$, whereas that in (d) is LMB one for $g^{2}=0$ only, since there is no third-neighbour couplings. In all cases grey and black full circles denote spins from subsets $A$ and $B$, respectively. Solid lines denote exchange integrals greater than or equal to the threshold $g^{2}$, whereas those with $J \leq g^{2}$ are depicted by dashed lines. Since $g^{2}=0$ in the case (d) then it illustrates an octanuclear antiferromagnetic ring with ferromagnetic couplings of the second-neighbours

\subsection{Remarks}

It was not stated by the authors, but was pointed out in Ref. [19] that the proof presented in Ref. [5] is correct when the appropriate graph is connected [37], i.e. there exists a path connecting any two vertices. In the other words, for any pair $\{j, k\}$, there exits at least one $s$-element sequence $\left(s>0, l_{0} \equiv j, l_{s} \equiv k\right)$

$$
J_{j l_{1}}, J_{l_{1} l_{2}}, J_{l_{2} l_{3}}, \ldots, J_{l_{s-1} k}
$$

with non-zero elements $J_{l_{r} l_{r+1}}$ for all $0 \leq r<s$. In the opposite case, there are two (or more) non-interacting

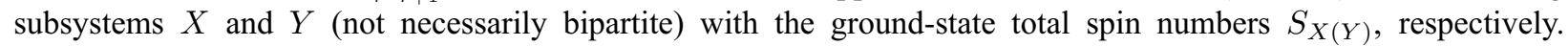
Hence, the whole system is highly degenerate with the total spin $S$ being any number in the range $\left(\mid S_{X}-\right.$ $\left.S_{Y} \mid, S_{X}+S_{Y}\right)$. Only for one of them equal to 0 , the ground-state of the system is a uniquely determined $S$-multiplet with $S=S_{X}$ (assuming $S_{Y}=0$ ) in the absence of accidental degeneration in the subsystems.

When $g^{2}=0$, then in each of the subsystems, only ferromagnetic exchange integrals are possible, so appropriate subsets constitute ferromagnetic components. Moreover, in this case, all cycles contains an even number of antiferromagnetic bonds, so a system in question is not geometrically frustrated [28,30,38, see also Fig. 2(d)]. However, for $g^{2}>0$ coexistence of LM-bipartiteness and (geometric) frustration is possible (see Figs. 2(a)-(c) and more detailed discussion in Refs. [29,30,32]). Even more, since systems considered are complete (and therefore connected for $g^{2}>0$ ) then geometric frustration is surely present, since there is at least one antiferromagnetic coupling in one of the subsystems. If such bonds do not exist then $g^{2}=0$ may be assumed and, henceforth, the geometric frustration is absent.

In the paper by Lieb and Mattis [5], there is no formal exclusion of empty sets, but assuming, for example, $B=\emptyset$ trivial results are received (see Sec. 3 below). It is only worth to mention that in such a case for each pair $\{j, k\}$, there exists at least one sequence (3) with all exchange integrals being negative. Hence, the system cannot be decomposed, but it is not necessarily ferromagnetic (cf. Ref. [5]).

\section{The Lieb-Mattis theorem and its applications}

\subsection{The theorem}

For the LMB system the numbers $S_{A(B)}$ and their absolute difference are introduced:

$$
S_{A}=\sum_{j \in A} s_{j}, S_{B}=\sum_{k \in B} s_{k}, \mathcal{S}=\left|S_{A}-S_{B}\right|
$$

Lieb and Mattis proved that the ground state of the Hamiltonian (1) belongs at most to total spin $S=\mathcal{S}$. Hence, the absolute difference is the upper limit of the ground-state total spin: $S_{0} \leq \mathcal{S}$. Let $E_{\min }(S)$ denotes the minimum energy of states belonging to the total spin $S$. The LMF says that these energies form an increasing sequence, i.e.:

$$
E_{\min }(S)>E_{\min }(S-1) \text { for all } S>\mathcal{S} .
$$

This is referred to as the Lieb-Mattis level order (LMLO) and, introducing differences:

$$
\Delta_{S}=E_{\min }(S)-E_{\min }(S-1),
$$




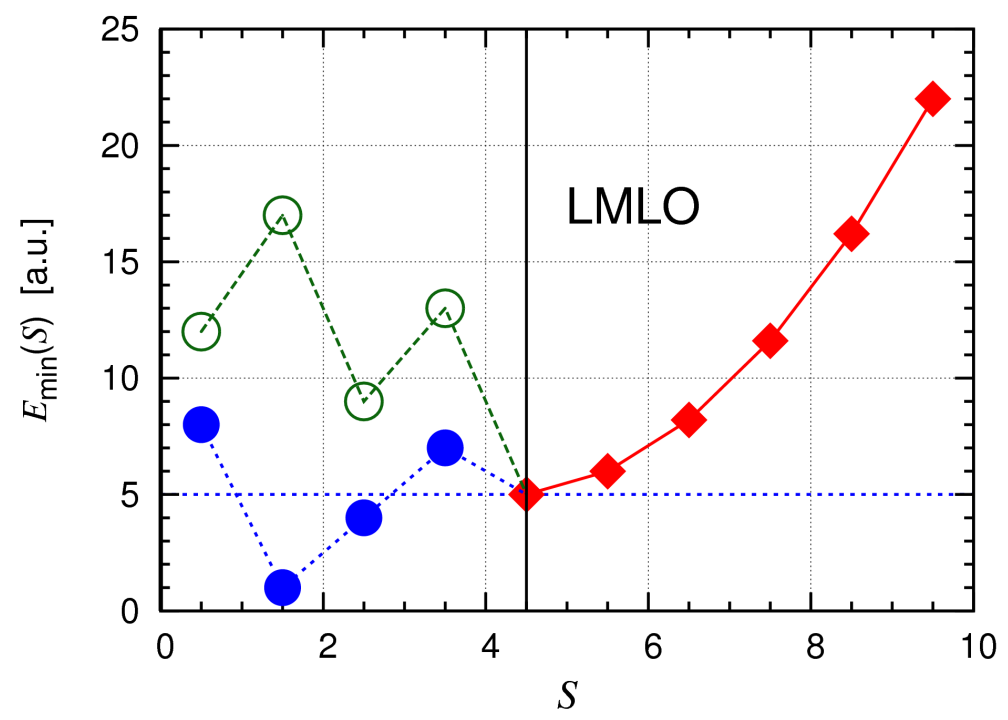

FIG. 3. Illustration of the Lieb-Mattis level order in LM-bipartite systems. For the sake of simplicity it is assumed that the Landé band for $S \geq \mathcal{S}$ (full squares) is parabolic. Full and empty circles correspond to $g^{2}>0$ and $g^{2}=0$, respectively. Lines are a guide for the eye

can be reformulated as: $\Delta_{S}$ are positive for all $S>\mathcal{S}$. This effect is observed in many quantum spins systems and it is said that eigenstates with energies $E_{\min }(S)$ form the so-called Landé band [39,40]. In the special case of non-frustrated systems, i.e. for $g^{2}=0$, a stronger constraint can be proved [5]:

$$
E_{\min }(S)>E_{\min }(\mathcal{S}), \text { for } S<\mathcal{S},
$$

which unambiguously determines $S_{0}=\mathcal{S}$ (see Fig. 3). It has to be emphasized that the LMT says nothing about ordering of levels with $S<\mathcal{S}$. A general formulation of the LMT is also referred to as the 'weak form' with the 'strong form' assigned to the case $g^{2}=0$.

If, by chance, there are a few possible LM-bipartitions, with different threshold $g^{2}$ probably, then the most restrictive one, i.e. this with the least $\mathcal{S}$, is applied. If the case $B=\emptyset$ was allowed, then the trivial result would be obtained $S_{0} \leq S_{\max }=\sum_{j=1}^{n} s_{j}$, with the equality sign for purely ferromagnetic systems only ( $g^{2}=0$ would force all non-zero weights $J_{j k}$ to be negative in this case). Assuming systems in Fig. 2 to be homogeneous ( $s_{j}=s$ for all $1 \leq j \leq n$ ) the following constraints can be easily determined: (a) $S_{0} \leq s$, (b) and (d) $S_{0}=0$, and (c) $S_{0} \leq 5 s$. Other examples can be found in Refs. [28-30,32,41].

A very important example of LMB systems is the so-called sublattice Hamiltonian or the rotational band model $[39,42-44]$. Spins are divided into subsets $A$ and $B$ with all pairs $\{j, k\}, j \in A, k \in B$, coupled antiferromagnetically with the same magnitude $J>0$, so:

$$
H=J \boldsymbol{S}_{A} \cdot \boldsymbol{S}_{B}, \quad \text { where } S_{A(B)}=\sum_{j \in A(B)} \boldsymbol{s}_{j} .
$$

This system is LM-bipartite with any $0 \leq g^{2} \leq J$, so the least value $\left(g^{2}=0\right)$ yields $S_{0}=\left|S_{A}-S_{B}\right|$ and the corresponding graph is complete bipartite one.

\subsection{Remarks about the proof}

The original proof in Ref. [5] was decomposed into two parts: (i) a detailed discussion on the case $g^{2}=0$ and (ii) a short comment on the general case, so this paper goes along this way. The most crucial is a canonical transformation:

$$
s_{j}^{x} \rightarrow-s_{j}^{x}, \quad s_{j}^{y} \rightarrow-s_{j}^{y}, \quad s_{j}^{z} \rightarrow+s_{j}^{z}
$$

for all spins in a chosen subsystem, say $j \in A$. It yields all off-diagonal entries to be non-positive [5, 19]. Further analysis may be done repeating Lieb and Mattis's reasoning or the Perron-Frobenius theorem (PFT) may be invoked (see below and Ref. [19]). 


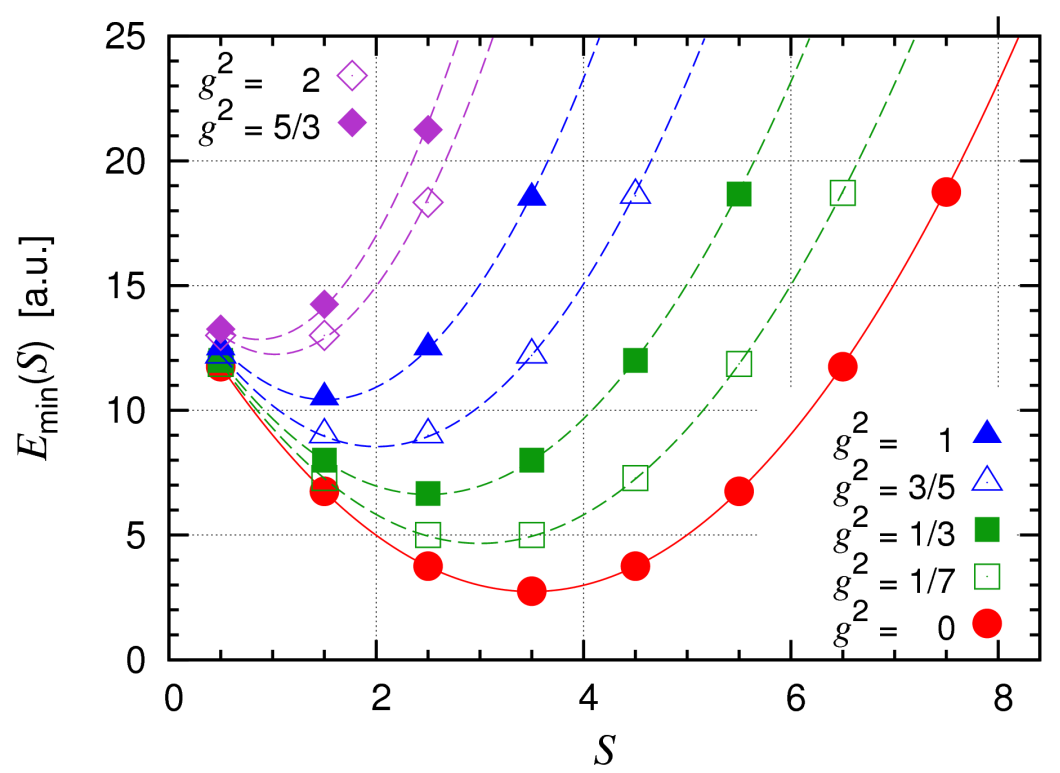

FIG. 4. The energies $E_{\min }(S)$ (the Landé band) for fictitious LM-bipartite Hamiltonians $H(g)=$ $g^{2} S^{2}+S(S-7)+15$. Symbols are defined in the legend and lines are a guide for the eye

When a Hamiltonian, commuting with $\boldsymbol{S}^{2}$, is LM-bipartite for $g^{2}>0$ then, it is transformed to:

$$
H^{\prime}=H-g^{2} \boldsymbol{S}^{2}
$$

which determines the LMB system with $g^{\prime}=0$. Since usually the threshold $g^{2}$ can be chosen in (infinitely) many ways, it must be done carefully - the Hamiltonian $H^{\prime}$ has to satisfy all conditions stated in the previous section, among others the corresponding bipartite weighted graph has to be connected and complete. This transformation does affect the upper limit given by $\mathcal{S}$ (the partition into sets $A$ and $B$ is not modified) and the LMLO for $S>\mathcal{S}$ is preserved. However, the inequality (7) does not hold, in a general case. Modifying a bit Eq. (10) one may introduce:

$$
H(g)=H^{\prime}+g^{2} \boldsymbol{S}^{2}
$$

and observe changes in energies $E_{\min }(S)$ for different values of $g^{2}$. A simple example is presented in Fig. 4, where $H^{\prime}=S(S-7)+15$ is assumed. The ground-state total spin $S_{0}$ decreases for increasing $g^{2}$, yielding the sequence $S_{0}=7 / 2,5 / 2,3 / 2,1 / 2$ with 'critical' values of $g^{2}=1 / 7,3 / 5$, and $5 / 3$ (at this points the ground-state level is formed by two $S$-multiplets with different total spin number $S$, so the degenerate frustration may be observed [45]).

However, the second (increasing) part of the universal sequence discussed in Refs. [29,30] cannot be explained in this way. It is important to emphasize that this sequence is received when the energies $E_{\min }(S)$ for $S<\mathcal{S}$ are ordered, what constitutes the special case. In a general case, different values of $S_{0}$ may appear in any order (cf. Fig. 3).

To end this section, the relation between the two theorems, Lieb-Mattis and Perron-Frobenius, is discussed. The latter one (PFT) is presented after Ref. [19]. Let $A$ be a square matrix of size $N>1$ with all entries being non-negative real numbers. If there exits $p \geq 1$ that $A^{p}$ has strictly positive entries, then, with $\Lambda$ being a set of all eigenvalues of $A$ :

(1) $\lambda_{0}=\max _{\lambda \in \Lambda}|\lambda| \in \Lambda$ and it is simple (non-degenerate), i.e. it is a simple root of $\operatorname{det}\left(A-\lambda \mathbb{I}_{N}\right)=0$, where $\mathbb{I}_{N}$ is the unit matrix;

(2) for any other $\lambda \in \Lambda,|\lambda|<\lambda_{0}$;

(3) the eigenvector of $A$ associated to $\lambda_{0}$ has strictly positive coordinates.

These conditions are satisfied, for example, for the operator $S^{2}$, so a vector with positive coordinates is associated to $S_{\max }$ in each subspace with given total magnetization $M$.

To use the PFT in the proof of the LMT, it is necessary to perform such modification of the Hamiltonian matrix that the above requirements are satisfied. The first step is done with the transformation (9). Since the minimum is what ones looks for, then the sign of this matrix is changed. The eigenvalues $E_{\min } \leq E \leq E_{\max }$ of the Hamiltonian (1) satisfy, as a rule, $E_{\min }<0, E_{\max }>0$, and $\left|E_{\min }\right|>E_{\max }$, so these two steps should be enough. In 
the other case, to ensure that $E_{\min }$ and the corresponding eigenvector are determined properly, a shift may be done, i.e. a matrix $\gamma \mathbb{I}_{N}$ with enough large $\gamma>0$ is added to obtain the matrix $A$. The connectivity of the corresponding graph guarantees that for some $p \geq 1$ all matrix elements of $A^{p}$ are strictly positive.

\section{Open problems}

The LMT, with the proof sketched in the previous section, explains many features observed in investigated models of molecular nanomagnets. However, there are facts, which cannot be assigned to this theorem in the present form. It has been already mentioned above that the second part of the total spin sequence discussed in Refs. $[29,30]$ cannot be explained by the simple analysis illustrated in Fig. 4. Moreover, in the case of ring-shaped molecules studied some features go beyond the properties given by the LMT. E.g., the differences (6) are not only positive, but also form an increasing sequence (see Fig. 5):

$$
\Delta_{S}>\Delta_{S-1}, \text { for all } S>S_{0}
$$

note that is true also for $S_{0}<\mathcal{S}$ [30,32]. Even if this fact is not directly mentioned, it is confirmed by magnetization steps presented (see, e.g., Refs. [34,46]).

These phenomena are clearly seen considering a very simple model of a heterogeneous centered square (see the inset in Fig. 5) with the Hamiltonian:

$$
H_{\square}=J_{1}\left(s_{1}+s_{3}\right) \cdot\left(s_{2}+s_{4}\right)+J_{2} s_{0} \cdot\left(s_{1}+s_{2}+s_{3}+s_{4}\right) .
$$

To keep this system symmetric, it is postulated that $s_{j}=s$ for $1 \leq j \leq 4$ with $s_{0}=s^{\prime}$ in a general case. Introducing intermediate spins:

$$
\boldsymbol{S}_{A}=s_{1}+s_{3}, \quad \boldsymbol{S}_{B}=s_{2}+s_{4}, \quad \boldsymbol{S}_{\square}=\boldsymbol{S}_{A}+\boldsymbol{S}_{B}, \quad \boldsymbol{S}=\boldsymbol{s}_{0}+\boldsymbol{S}_{\square}
$$

and assuming $J_{1}=1, J_{2}=\alpha J_{1}$ it can be rewritten as:

$$
H_{\square}=\boldsymbol{S}_{A} \cdot \boldsymbol{S}_{B}+\alpha \boldsymbol{s}_{0} \cdot \boldsymbol{S}_{\square},
$$

so its eigenvalues can be exactly determined with simple algebra for all values of the spin numbers $0 \leq S_{A, B} \leq 2 s$, $0 \leq S_{\square} \leq 4 s, 0 \leq S \leq 4 s+s^{\prime}$ and the parameter $\alpha \in \mathbb{R}$ (cf. Refs. [30,34,43]). Some ranges of the parameter $\alpha$ can be clearly distinguished:

(1) $\alpha<0$ : The system is not LMB, so the constraint $S_{0} \leq 4 s+s^{\prime}$ is applicable only.

(2) $\alpha=0$ : Two non-interacting systems are present, but one of them has $S_{\square}=0$, so it has to be $S_{0}=s^{\prime}$ with any accidental degeneration.

(3) $0<\alpha<1$ : there are two possibilities:

(a) To take $\alpha<g^{2}<1$ and consider the system as one-component with $S_{0} \leq 4 s+s^{\prime}$.

(b) To consider a bipartition $A=\{1,3\}, B=\{0,2,4\}$ with $g^{2}=\alpha$. However:

$$
H_{\square}^{\prime}=(1-\alpha) \boldsymbol{S}_{A} \cdot \boldsymbol{S}_{B}-\alpha\left(\boldsymbol{s}_{1} \cdot \boldsymbol{s}_{3}+\boldsymbol{s}_{2} \cdot \boldsymbol{s}_{4}\right),
$$

so two non-interacting subsystems, as in the case (2) above, are obtained, and therefore, formally, the LMT cannot be applied.

(4) $\alpha=1$ : It is a very particular point. Assuming $g^{2}>1$ yields $H^{\prime}$ describing the single ferromagnetic component, whereas $g^{2}=1$ leads to two ferromagnetic components $\left(A^{\prime}=\{1,3\}, B^{\prime}=\{2,4\}\right)$ and isolated spin $s_{0}$.

(5) $\alpha>1$ : The domain of the LMT is reached, at last. The system is LMB for any $1 \leq g^{2} \leq \alpha$ and for the least value $g^{2}=1$ one has:

$$
H^{\prime}=-\left(\boldsymbol{s}_{1} \cdot \boldsymbol{s}_{3}+\boldsymbol{s}_{2} \cdot \boldsymbol{s}_{4}\right)+(\alpha-1) \boldsymbol{s}_{0} \cdot \boldsymbol{S}_{\square}
$$

with obvious LM-bipartition $A^{\prime}=\{1,2,3,4\}, B^{\prime}=\{0\}$, so $\mathcal{S}=\left|4 s-s^{\prime}\right|$.

The results obtained for $s=1, s^{\prime}=2$ are presented in Fig. 5. It is evident that the LMLO is present for all $\alpha \in \mathbb{R}$, even with the stronger constraint given by Eq. (11). Also the sequence of the ground-state total spin $S_{0}(\alpha)$ (see the inset in Fig. 5) is consistent with the previous discussion [29, 30, 32, 47, see also Fig. 4], though $g^{2}$ can be assumed constant here. Analogous results were also obtained for the isosceles triangle [29,30].

All the above features follow the special properties of ring-shaped molecules investigated and can be explained by 'specialized' forms of the LMT or more detailed studies, including careful analysis of the energy spectrum for $S<\mathcal{S}$ and discussion on the special cases: two isolated subsystems or one-component systems (cf. Ref. [19]). It is very likely that in some cases such considerations will allow to apply the PFT in its original form. However, the same properties have been confirmed by exact diagonalization calculations for larger systems $[29,30,32,46]$, which, in general, are not LMB systems. In such cases the crucial transformation (9), performed for any decomposition into 


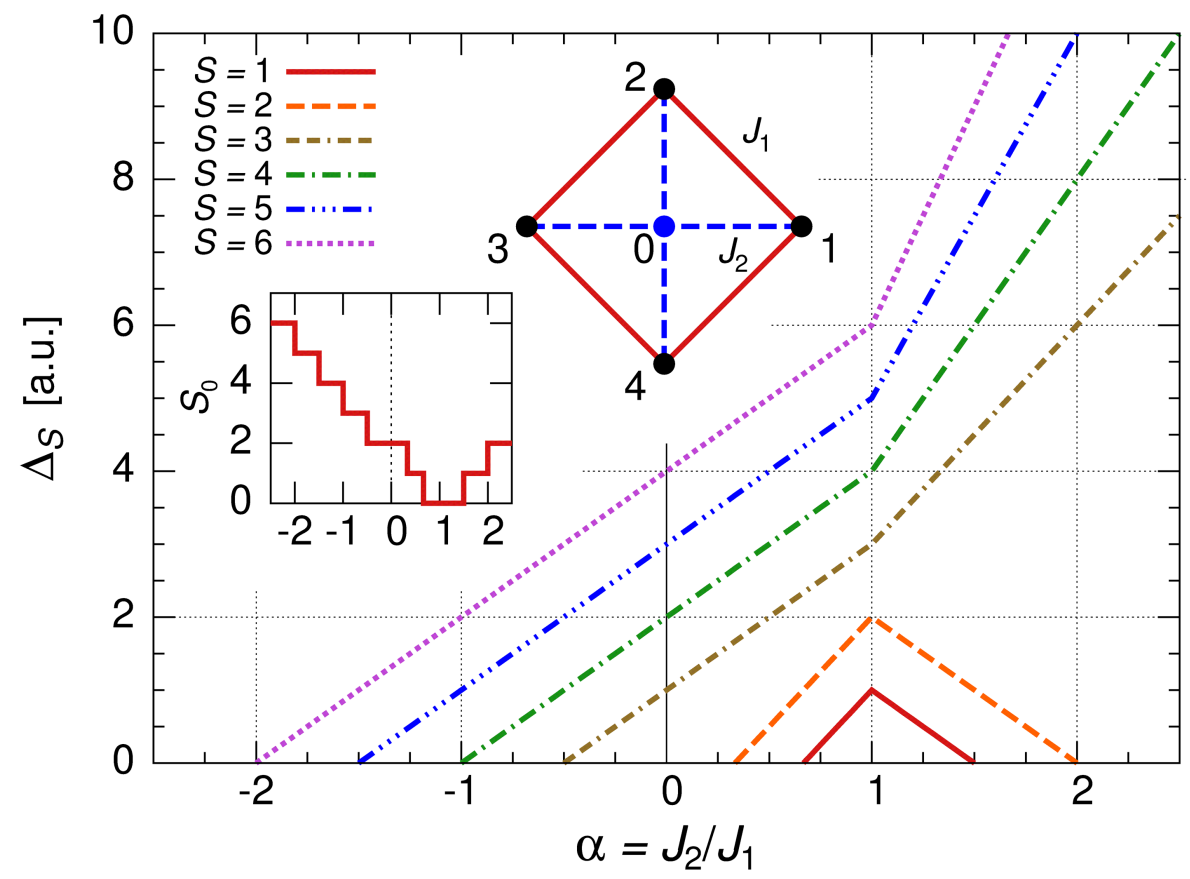

FIG. 5. The LMLO in a heterogeneous centred square presented in the inset $\left(s_{0}=2, s_{1}=s_{2}=\right.$ $s_{3}=s_{4}=1, J_{1}>0$ ). Irrelevant segments (with $S<S_{0}$ ) are omitted. Zeros of $\Delta_{S}$ are placed at critical values $\alpha_{\mathrm{c}}^{(k)}=-2,-3 / 2,-1,-1 / 2,1 / 3,2 / 3,3 / 2,2$ (for $1 \leq k \leq 8$, respectively), where the ground-state total spin number $S_{0}$ is changed (see the inset)

two non-empty subsystems, leaves some negative entries in the corresponding Hamiltonian matrices. It is expected that among a huge number of works on some generalizations of the PFT, at least some of them, e.g. [25-27], will provide new tools and ways of reasoning, which will help in generalization of the LMT to larger systems which, already, show main features of the LMB ones.

\section{Conclusion and final remarks}

The Lieb-Mattis theorem has been applied to many quantum spin systems and considered and generalized in various ways. However, the LMB systems with $g^{2}>0$ have not been carefully analyzed, since they appear under exceptional conditions. Progress in synthesis of molecular nanomagnets has shown that the coexistence of geometric frustration and LM-bipartition is possible and this observation has put forward interest in a general ('weak') form of the LMT. As it has been discussed above there are two main questions. At first, some features not included in the theorem itself, like the universal sequences of the ground-state total spin or the LMLO with the stronger condition (11), have to be investigated and explained. Secondly, the domain of the LMB should be thoroughly studied and necessary modifications should be done to include, for example, the whole range of the parameter $\alpha$ in Fig. 5 or larger, not LM-bipartite in general, systems. It can be presumed that a part of these problems can be solved applying the Perron-Frobenius theorem in its standard version, but in some cases generalizations of the PFT have to be taken into account.

Single-ion anisotropy plays the crucial role in investigations of single-molecule magnets and in many cases of anisotropic models some features analogous to LMLO are also observed [33,35,36,48,49]. However, in such cases the total spin is not a good quantum number, so the LMLO should be rather related to the total magnetization. Sometimes, for relatively small anisotropy parameters it is assumed that the total spin is, loosely speaking, 'an approximated good quantum number' and energy levels are labelled with $S$, even in the presence of anisotropy [40]. Such approach stands in contradictions to rigorous mathematical considerations presented above. Moreover, it was shown that such approximation can be accepted for levels with extreme energies, since they are quite stable, but there are abrupt changes of mean values of the total spin number for levels with intermediate energies [50,51]. On the other hand, it has to be carefully studied whether this is an intrinsic effect or it simply follows splitting of $S$-multiples observed in the isotropic regime. It should be emphasized that subspaces with a fixed magnetization are very important in the proof of the LMT, then rhombic anisotropy has to be excluded. 


\section{Acknowledgements}

The author would like to thank Grzegorz Kamieniarz for illuminating discussions and to Igor Yu. Popov for invitation to the MCQTN 2017 conference. Both of them, in different ways, have influenced the author to careful considerations of the Lieb-Mattis theorem, its assumptions, proofs and applications. Numerical confirmation of the results presented in this paper has been impossible without invaluable help of Michał Antkowiak.

\section{References}

[1] Gatteschi D., Sessoli R., Villain J. Molecular Nanomagnets. Oxford University Press, Oxford, 2006.

[2] Winpenny R. E. P. Molecular Cluster Magnets. World Scientific Publ. Co., Singapore 2012.

[3] Molecular Nanomagnets and Related Phenomena, (Ed.: S. Gao), Springer, Berlin-Heidelberg 2015.

[4] Miller J., Gatteschi D. Molecule-based magnets. Chem. Soc. Rev., 2011, 40 (6), P. 3053-3368.

[5] Lieb E., Mattis D. Ordering energy levels of interacting spin systems. J. Math. Phys., 1962, 3, P. 749-751.

[6] Lieb E., Schultz T., Mattis D. Two soluble models of an antiferromagnetic chain. Annals of Physics, 1961,16, P. $407-466$.

[7] Buican M., Gromov A. Anyonic chains, topological defects, and conformal field theory. Commun. Math. Phys., 2017, 356, P. 1017-1056.

[8] Rajak A., Nag T. Survival probability in a quenched Majorana chain with an impurity. Phys. Rev. E, $2017,96,022136$.

[9] Nevado P., Fernández-Lorenzo S., Porras D. Topological edge states in periodically driven trapped-ion chains. Phys. Rev. Lett., 2017, 119, 210401.

[10] Cheng M., Zaletel M., et al. Translational symmetry and microscopic constraints on symmetry-enriched topological phases: A view from the surface. Phys. Rev. X, 2016, 6, 041068.

[11] Ruppeiner G., Bellucci S. Thermodynamic curvature for a two-parameter spin model with frustration. Phys. Rev. E, 2015, 91, 012116.

[12] Miyao T. Quantum Griffiths inequalities. J. Stat. Phys., 2016, 164, P. 255-303.

[13] Tylutki M., Astrakharchik G. E., Recati A. Coherent oscillations in small Fermi-polaron systems. Phys. Rev. A, $2017,96,063603$.

[14] Pan L., Liu Y., et al. Exact ordering of energy levels for one-dimensional interacting Fermi gases with $S U(N)$ symmetry. Phys. Rev. $B$, 2017, 96, 075149.

[15] Oshikawa M. Commensurability, excitation gap, and topology in quantum many-particle systems on a periodic lattice. Phys. Rev. Lett., 2000, 84, P. $1535-1538$

[16] Nachtergaele B., Sims R. Commun. Math. Phys., 2007, 276, P. 437-472.

[17] Zaletel M.P., Vishwanath A. Constraints on topological order in Mott insulators. Phys. Rev. Lett., $2015,114,077201$.

[18] Nomura K., Morishige J., Takaichi I. Extension of the Lieb-Schultz-Mattis theorem. J. Phys. A: Math. Theor., $2015,48,375001$.

[19] Nachtergaele B., Spitzer W., Starr S. Ferromagnetic ordering of energy levels. J. Stat. Phys., 2004, 116, P. 719-738.

[20] Perron O. Zur Theorie der Matrizen. Math. Ann., 1907, 64, P. 248-263.

[21] Frobenius G. Über Matrizen aus nicht negativen Elementen. S.-B. Preuss Acad. Wiss., Berlin 1912, pp. $456-477$.

[22] Simon B. The Statistical Mechanics of Lattice Gases, Vol. 1, Princeton Univ. Press, 1993.

[23] MacCluer C.R. The many proofs and applications of Perron's theorem. SIAM Review, 2000, 42, P. $487-498$.

[24] Hawkins T. Continued fractions and the origins of the Perron-Frobenius theorem. Arch. Hist. Exact Sci., 2008, 62, P. 655-717.

[25] Rump S.M. Theorems of Perron-Frobenius type for matrices without sign restrictions. Lin. Alg. Appl., 1997, 266, P. 1-42.

[26] Tarazaga P., Raydan M., Hurman A. Perron-Frobenius theorem for matrices with some negative entries. Lin. Alg. Appl., 2001, 328, P. 57-68.

[27] Noutsos D. On Perron-Frobenius property of matrices having some negative entries. Lin. Alg. Appl., 2006, 412, P. 132-153.

[28] Schnack J. Effects of frustration on magnetic molecules: a survey from Olivier Kahn until today. Dalton Trans., 2010,39 , P. 4677-4686.

[29] Kamieniarz G., Florek W., Antkowiak M. Universal sequence of ground states validating the classification of frustration in antiferromagnetic rings with a single bond defect. Phys. Rev. B, 2015, 92, 140411.

[30] Florek W., Antkowiak M., Kamieniarz G. Sequences of ground states and classification of frustration in odd-numbered antiferromagnetic rings. Phys. Rev. B, 2016, 94, 224421.

[31] Schnack J. Frustration effects in antiferromagnetic molecules: The cuboctahedron. Polyhedron, 2009, 28, P. 1620-1623.

[32] Antkowiak M., Florek W., Kamieniarz G. Universal sequence of the ground states and energy level ordering in frustrated antiferromagnetic rings with a single bond defect. Acta Phys. Pol. A, 2017, 131, P. 890-892.

[33] Caciuffo R., Guidi T., et al. Spin dynamics of heterometallic $\mathrm{Cr}_{7} \mathrm{M}$ wheels $\mathrm{M}=\mathrm{Mn}, \mathrm{Zn}$, Ni) probed by inelastic neutron scattering. Phys. Rev. B, 2005, 71, 174407 .

[34] Furrer A., Krämer K.W., et al. Magnetic and neutron spectroscopic properties of the tetrameric nickel compound $\left[\mathrm{Mo}_{12} \mathrm{O}_{28}\left(\mu_{2}-\mathrm{OH}\right)_{9}\left(\mu_{3}-\right.\right.$ $\left.\mathrm{OH})_{3}\left\{\mathrm{Ni}\left(\mathrm{H}_{2} \mathrm{O}\right)_{3}\right\}_{4}\right] \cdot 13 \mathrm{H}_{2} \mathrm{O}$. Phys. Rev. B, 2010, 81, 214437.

[35] Antkowiak M., Kozłowski P., et al. Detection of ground states in frustrated molecular rings by in-field local magnetization profiles. Phys. Rev. B, 2013, 87, 184430 .

[36] Antkowiak M., Kucharski E., Kamieniarz G. Non-uniform coupling model of the frustrated chromium-based ring Cr. $\mathrm{Ni}$ EPJ Web of Conferences, 2014, 75, 05007.

[37] Wilson R.J. Introduction to Graph Theory, Adison Wesley Longman Ltd., 4th edition, London, 1996.

[38] Toulouse G. Theory of the frustration effect in spin glasses: I. Communications de Physique, 1977, 2, P. 115-119; Reprinted in Spin Glass Theory and Beyond (Eds.: Mézard M., Parisi G., Virasoro M.A.), World Scientific Publ. Co., Singapore, 1987, P. 99-103.

[39] Waldmann O. Spin dynamics of finite antiferromagnetic Heisenberg spin rings. Phys. Rev. B, $2001,65,024424$.

[40] Kamieniarz G., Kozłowski P., et al. Phenomenological modeling of molecular-based rings beyond the strong exchange limit: Bond alternation and single-ion anisotropy effects. Inorg. Chimica Acta, 2008, 361, P. 3690-3696.

[41] Sobocińska M., Antkowiak M., et al. New tetranuclear manganese clusters with $\left[\mathrm{Mn}_{3}^{\mathrm{II}} \mathrm{Mn}^{\mathrm{III}}\right]$ and $\left[\mathrm{Mn}_{2}^{\mathrm{II}} \mathrm{Mn}_{2}^{\mathrm{III}}\right] \mathrm{metallic}^{\mathrm{I}}$ cores exhibiting low and high spin ground state. Dalton Trans., 2016, 45, P. 7303-7311.

[42] Schnack J., Luban M., Modler R. Quantum rotational band model for the Heisenberg molecular magnet Mo ${ }_{72}$ Fe $_{30}$. Europhys. Lett., 2001, 56, P. 863-869. 
[43] Florek W., Kaliszan L.A., Kunert H.W., Machatine A.G.J. Small antiferromagnetic spin systems - sublattice Hamiltonians. Physica B, 2010, 405, P. 3811-3817.

[44] Florek W., Kaliszan L.A. Small antiferromagnetic spin systems: Just beyond the rotational band model. Acta Phys. Pol. A, 2015, 127, P. $330-332$.

[45] Kahn O. Competing spin interactions and degenerate frustration for discrete molecular species. Chem. Phys. Lett., 1997, 265, P. $109-114$.

[46] Sharples J.W., Collison D., et al. Quantum signatures of a molecular nanomagnet in direct magnetocaloric measurements. Nature Commun., 2014, 5, 5321.

[47] Ako A.M., Waldmann O., et al. Odd-numbered Fe ${ }^{\mathrm{III}}$ complexes: Synthesis, molecular structure, reactivity, and magnetic properties. Inorg. Chem., 2007, 46, P. 756-766.

[48] Kozłowski P, Antkowiak M., Kamieniarz G. Frustration signatures in the anisotropic model of a nine-spin $s=3 / 2$ ring with bond defect. J. Nanopart. Res., 2011, 13, P. 6093-6102.

[49] Baker M.L., Guidi T., et al. Spin dynamics of molecular nanomagnets unravelled at atomic scale by four-dimensional inelastic neutron scattering. Nature Phys., 2012, 8, P. 906-911.

[50] Florek W., Jaśniewicz-Pacer K, Kaliszan L. A., Kamieniarz G. Single-ion anisotropy in a four-spin system: mixing of $S$-states. Cen. Eur. J. Chem., 2009, 7, P. 211-214.

[51] Florek W., Kaliszan L.A., Jaśniewicz-Pacer K., Antkowiak M. Numerical analysis of magnetic states mixing in the Heisenberg model with the dihedral symmetry. EPJ Web of Conferences, 2013, 40, 14003. 